Gazi University
Journal of Science
$\mathrm{http} / /$ dergipark.gov.tr/gujs

\title{
Some Properties of a Third Order Partial Recurrence Relation
}

\author{
Anthony G. SHANNON ${ }^{1, *}$ (D) , Omur DEVECI ${ }^{2}$ \\ ${ }^{I}$ The University of New South Wales, Warrane College, Kensington, NSW 2033, Australia \\ ${ }^{2}$ Kafkas University, Faculty of Science and Letters, Department of Mathematics, 36100 Kars, Turkey
}

\section{Highlights}

- This paper considers some of the properties of sequences $\left\{v_{m, n}\right\}$.

- The relationship between a generalized continued fraction and arbitrary sequence explores.

- This paper considers partial recurrence relation related to the third order recurrence relation.

\section{Article Info}

Received: 16 July 2020 Accepted: 12 Jan 2021

\section{Keywords}

Fibonacci numbers

Tribonacci numbers

Partial quotients

Euclidean algorithm

\begin{abstract}
This paper explores a connection between third order recursive sequences and generalized continued fractions by analogy with second order recursive sequences and ordinary twodimensional continued fractions. It does this with a partial recurrence relation which is related to the original third order recurrence relation, and raises a related conjecture.
\end{abstract}

\section{INTRODUCTION}

We define 3 basic $3^{\text {rd }}$ order linear recursive sequences $\left\{t_{m, n}\right\} m=0,1,2$ by initial values for $n=0,1,2$ and the $3^{\text {rd }}$ order linear homogeneous recurrence relation

$$
t_{m, n}=P_{1} t_{m, n-1}+P_{2} t_{m, n-2}+P_{3} t_{m, n-3}, n \geq 3,
$$

in which the $P_{j}$ are arbitrary integers so that (1) is essentially analogous to Horadam's generalized sequence for his $2^{\text {nd }}$ order $\left\{w_{n}\right\}$ [1]. For later convenience, we shall take the initial values as $t_{m, 0}=t_{m, 1}=t_{m, 2}=$ 1 when $P_{1}=0, P_{2}=P_{3}=1$, a Padovan sequence [2] - sequence A00031 in Sloane [3].

We also define related arrays of sequences $\left\{v_{m, n}\right\}$ for any integer $m$ by a partial recurrence relation

$$
v_{m, n}=v_{m-1, n-1}+P_{3-n} v_{m-1,2}, 0<m<3, n>0,
$$

with boundary conditions

$$
v_{m, 0}=P_{3} t_{n} \text { and } v_{0, n}=P_{3-n} .
$$

The purpose of this paper is to explore some of the properties of this array. 


\section{PARTIAL RECURRENCE RELATION}

We first show that the characteristic equation associated with (1) is related to the partial recurrence relation by

$$
x^{3}=\sum_{n=0}^{2} P_{3-n} x^{n} \rightarrow x^{m+3}=\sum_{n=0}^{2} v_{m, n} x^{n}
$$

the proof of which follows by induction on $m$.

When $m=0$,

$$
\begin{aligned}
\sum_{n=0}^{2} v_{0, n x^{n}} & =\sum_{n=0}^{2} P_{3-n} x^{n} \\
& =x^{3} .
\end{aligned}
$$

When $m=1$,

$$
\begin{aligned}
\sum_{n=0}^{2} v_{1, n} x^{n} & =\sum_{n=0}^{2}\left(v_{0, n-1}+P_{3-n} v_{0,2}\right) x^{n} \\
& =x \sum_{n=0}^{2} v_{0, n-1} x^{n-1}+v_{0,2} \sum_{n=0}^{2} P_{3-n} x^{n} \\
& =x \sum_{n=0}^{1} v_{0, n} x^{n}+v_{0,2} x^{3} \\
& =x \sum_{n=0}^{2} v_{0, n} x^{n} \\
& =x \sum_{n=0}^{2} P_{3-n} x^{n} \\
& =x^{4} .
\end{aligned}
$$

Assume the result is true for $m=2,3, \ldots, s$. Then

$$
\begin{aligned}
x^{s+4} & =\sum_{n=0}^{2} v_{s, n} x^{n+1} \\
& =v_{s, 2} x^{3}+\sum_{n=0}^{1} v_{s, n} x^{n+1} \\
& =v_{s, 2} x^{3}+\sum_{n=0}^{2} v_{s, n-1} x^{n} \\
& =v_{s, 2} x^{3}+\sum_{n=0}^{2}\left(v_{s+1, n}-P_{3-n} v_{s, 2}\right) x^{n}
\end{aligned}
$$




$$
\begin{aligned}
& =v_{s, 2} x^{3}-v_{s, 2} \sum_{n=0}^{2} P_{3-n} x^{n}+\sum_{n=0}^{2} v_{s+1, n} x^{n} \\
& =v_{s, 2}\left(x^{3}-P_{3}-P_{2} x-P_{1} x^{2}\right)+\sum_{n=0}^{2} v_{s+1, n} x^{n} \\
& =\sum_{n=0}^{2} v_{s+1, n} x^{n} .
\end{aligned}
$$

\section{APPLICATION}

We now apply this to the case where $P_{1}=0, P_{2}=P_{3}=1$, which Hildebrand [4] used as an example in oldfashioned numerical analysis; that is, for

$$
x^{6}=\sum_{n=0}^{2} v_{3, n} x^{n}=1+2 x+x^{2} .
$$

We also have from (4) that

$$
\begin{aligned}
f(3) & =x^{3} \\
& =x+1
\end{aligned}
$$

which is, in effect, the auxiliary equation for the corresponding third order Padovan sequence, some properties of which have been developed in [5-9]. If we apply the result (4), we see initially that

$$
\begin{aligned}
& f(3)=x+1 \\
& f(4)=x^{2}+x \\
& f(5)=x^{2}+2 x+1
\end{aligned}
$$

and so on by using (4) until

$$
f(48)=170625 x^{2}+226030 x+128801
$$

and

$$
f(49)=226030 x^{2}+299426 x+170625
$$

which conforms with Gnanadoss [10], to find that if we use $x=1.3$ as an approximation of the dominant root of $x^{3}-x-1=0$, then a better approximation with the Bernoulli iteration can be given by

$$
\frac{f(49)}{f(48)}=1.324717973 . .
$$

which agrees with Hildebrand.

\section{GENERALIZED CONTINUED FRACTIONS}

Fibonacci numbers have many connections with ordinary continued fractions both pure (complex analysis and number theory) [11,12], and applied (linguistics and numerical analysis) [13]. Here we consider a plausible case for how a generalized continued fraction can be related to arbitrary order sequences. Previous work (for example $[14,15]$ ) has tended to focus on going from the continued fraction algorithm to the recurrence relation or difference equation, whereas we are attempting to go in the opposite direction.

From (2) we can write, both formally and more generally, that for $j=0,1,2,(j=1$ is the case in (2)): 


$$
v_{3-j, n}=v_{2-j, n-1}+P_{j} v_{m-1,2}, \quad n>0,
$$

and

$$
v_{2, n}=v_{1, n-1}+P_{1} v_{2,2}
$$

so that if we put

$$
a_{j}^{(n)}=\frac{v_{j-1, n}}{v_{2, n}}
$$

then

$$
\frac{v_{2-j, n-1} / v_{2, n}}{v_{2, n+1} / v_{2, n}}=\frac{\left(v_{2-j, n} / v_{2, n}\right)-P_{j}}{\left(v_{1, n} / v_{2, n}\right)-P_{j}}
$$

and if

$$
b_{j}^{(n)}=(-1)^{j} P_{j},
$$

then

$$
a_{j-1}^{(n+1)}=\frac{a_{j}^{(n)}-b_{j}^{(n)}}{a_{1}^{(n)}-b_{1}^{(n)}}
$$

if we set $a_{0}^{(n)}=1, \forall n$.

An ordinary periodic continued fraction is an irrational root of a quadratic equation with irrational roots; in particular, the convergents satisfy second order linear recurrence relations with suitable initial conditions with the $n^{\text {th }}$ convergent

where

$$
\frac{p_{n}}{q_{n}}=\frac{x_{1}^{n+2}-x_{2}^{n+2}}{x_{1}^{n+1}-x_{2}^{n+1}}
$$

$$
p_{n+1}=a_{n} p_{n}+p_{n-1},
$$

and

$$
q_{n+1}=a_{n} q_{n}+q_{n-1}
$$

For $k$ a non-negative integer and $a_{i}^{(k)} \in R, i=1,2, \ldots, n-1$, let [16]

$$
a^{(k)}=\left\lfloor a_{1}^{(k)}, a_{2}^{(k)}, \ldots, a_{n-1}^{(k)}\right\rfloor=a^{(k)} \in E_{n-1}
$$

and let

$$
f\left(a^{(k)}\right)=\left\lfloor b_{1}^{(k)}, b_{2}^{(k)}, \ldots, b_{n-1}^{(k)}\right\rfloor=b^{(k)} \in E_{n-1}
$$

with $a_{1}^{(k)} \neq b_{1}^{(k)}$, so that for $T: E_{n-1} \rightarrow E_{n-1}$

$$
a^{(k)} T=\left(a_{1}^{(k)}-b_{1}^{(k)}\right)^{-1}\left(a_{2}^{(k)}-b_{2}^{(k)}, a_{3}^{(k)}-b_{3}^{(k)}, \ldots, a_{n}^{(k)}-b_{n}^{(k)}\right)=a^{(k+1)}
$$

which is a Jacobi-Perron Algorithm (JPA) of the vector $a^{(0)}$ as a sequence $\left\{a^{(k)}\right\}$ of vectors in $E_{r-1}$ and Bernstein has demonstrated how this is a generalization of the continued fraction algorithm [16]. 


\section{CONCLUDING SUGGESTIONS}

This raises the related question that given the connection between the ordinary continued fraction and the Euclidean Algorithm, can the latter also be generalized along the following lines.

Instead of relating two integers, suppose we connect two pairs of integers $(a, b)$ and $(c, d)$ in steps analogous to the ordinary Euclidean Algorithm.

Suppose $c>a>b>d$, so that

$$
\begin{aligned}
(c, d) & =x_{1}(a, b)+\left(c-x_{1} a,-x_{1} b+d\right) \\
(a, b) & =x_{2}\left(c-x_{1} a,-x_{1} b+d\right)+\left(a-x_{2}\left(c-x_{1} a\right), b-x_{2}\left(-x_{1} b+d\right)\right) \\
\ldots & =x_{n}(u, v)+(-b,-a) .
\end{aligned}
$$

For example, consider the vector pair $(17,1)$ and $(65,0)$ :

$$
\begin{aligned}
(65,0) & =3(17,1)+(14,-3) \\
(17,1) & =1(14,-3)+(3,4) \\
(14,-3) & =4(3,4)+(2,-19) \\
(3,4) & =1(2,-19)+(1,23) \\
(2,-19) & =2(1,23)+(0,-65)
\end{aligned}
$$

in which from (6), the partial quotients are

$$
x_{1}=3, x_{2}=1, x_{3}=4, x_{4}=1, x_{5}=2 .
$$

In a sense (7) contains two Euclidean Algorithms, namely

$$
\begin{aligned}
65 & =3 \times 17+14 \\
17 & =1 \times 14+3 \\
14 & =4 \times 3+2 \\
3 & =1 \times 2+1 \\
2 & =2 \times 1+0
\end{aligned}
$$

and

$$
\begin{aligned}
65 & =2 \times 23+19 \\
23 & =1 \times 19+4 \\
19 & =4 \times 4+3 \\
4 & =1 \times 3+1 \\
3 & =3 \times 1+0 .
\end{aligned}
$$

The partial quotients in (8) are the same as those in (9) but in reverse order.

Conjecture: Consider the following equation

$$
t_{m, n}(i)=i P_{1} t_{m, n-1}-P_{2} t_{m, n-2}-i P_{3} t_{m, n-3}, n \geq 3
$$

and consider the initial values as the same. Then it should be possible to obtain new results using complex numbers among the equations in this paper. 


\section{CONFLICTS OF INTEREST}

No conflict of interest was declared by the authors.

\section{REFERENCES}

[1] Horadam, A.F., "Basic properties of a certain generalized sequence of numbers", The Fibonacci Quarterly, 3: 161-176, (1965).

[2] Deveci, O., Shannon, A.G., "Pell-Padovan-circulant sequences and their applications", Notes on Number Theory and Discrete Mathematics, 23 (3): 100-114, (2017).

[3] Sloane, N.J.A., "The On-Line Encyclopedia of Integer Sequences”, http://oeis.org, (1964).

[4] Hildebrand, F.B., Introduction to Numerical Analysis, McGraw-Hill, New York, 461, (1956).

[5] Feinberg, M., "New Slants", The Fibonacci Quarterly, 2: 223-227, (1964).

[6] Jarden, D., Recurring Sequences, Jerusalem: Riveon Lematika, 114, (1966).

[7] Shannon, A.G., Anderson, P.G., Horadam, A.F., "Properties of Cordonnier, Perrin and Van der Laan numbers", International Journal of Mathematical Education in Science and Technology, 37: 825-831, (2006).

[8] Shannon, A.G., Horadam, A.F., Anderson, P.G., "The auxiliary equation associated with the Plastic number", Notes on Number Theory and Discrete Mathematics, 12: 1-12, (2006).

[9] Shannon, A.G., Wong, C.K., "Some Properties of Generalized Third Order Pell Numbers", Congressus Numerantium, 201: 345-351, (2010).

[10] Gnanadoss, A.A., "Contracting Bernoulli's iteration and recurrence relations", The Mathematical Gazette, 44: 221-223, (1960).

[11] Anderson, P.G., Brown, T.C., Shiue, P.J.S., "A simple proof of a remarkable continued fraction identity", Proceedings of the American Mathematical Society, 123: 2005-2009, (1995).

[12] Anderson, P.G., "Notes and extensions for a remarkable continued fraction", The Fibonacci Quarterly, 55: 9-14, (2017).

[13] Van der Cruyssen, P., "Linear difference equations and generalized continued fractions", Computing, 22: 269-278, (1979).

[14] Szekeres, G., "Multidimensional Continued Fractions", Annales Universitatis Scientiarum Budapestinensis de Rolando Eötvös Nominatae Sectio Mathematica, 13: 113-140, (1970).

[15] Van der Cruyssen. P., “A continued fraction algorithm”, Numerische Mathematik, 37: 149156, (1981).

[16] Bernstein, L., The Jacobi-Perron Algorithm: Its Theory and Application (Lecture Notes in Mathematics 207), Berlin: Springer, Ch.2, (1971). 